Archives

7| 1991

Varia

\title{
La présence juive en Europe centrale àl'époque contemporaine. Invariants et spécificités
} nationales

\section{Victor Karady}

\section{OpenEdition}

1 Journals

\section{Édition électronique}

URL : http://journals.openedition.org/ccrh/2847

DOI : $10.4000 /$ ccrh. 2847

ISSN : 1760-7906

\section{Éditeur}

Centre de recherches historiques - EHESS

\section{Édition imprimée}

Date de publication : 15 avril 1991

ISSN : 0990-9141

Référence électronique

Victor Karady, « La présence juive en Europe centrale àl'époque contemporaine. Invariants et spécificités nationales », Les Cahiers du Centre de Recherches Historiques [En ligne], 7| 1991, mis en ligne le 18 mars 2009, consulté le 19 avril 2019. URL : http://journals.openedition.org/ccrh/2847 ; DOI : $10.4000 /$ ccrh. 2847

Ce document a été généré automatiquement le 19 avril 2019

Article L.111-1 du Code de la propriété intellectuelle. 


\title{
La présence juive en Europe centrale àl'époque contemporaine. Invariants et spécificités nationales
}

\author{
Victor Karady
}

\section{Les juifs, "centre-européens " par excellence de l'époque contemporaine. Aspects démographiques et culturels}

1 Si l'on identifie l'Europe centrale aux régions orientales de l'Europe marquées par le christianisme latin - comme l'ont proposé le médiéviste Jenö Szücs, et le politologue hongrois Istvàn Bibo - c'est à dire à la zone géographique qui s'étend entre les frontières historiques de la Russie et les États de l'Empire Germanique d'une part, et entre la mer du Nord et l'Adriatique d'autre part, les Juifs y occupent à l'époque contemporaine une position singulière à beaucoup d'égards :

2 - ils constituent le seul agrégat ethniquement distinct (ou défini comme distinct) qui ait été massivement présent dans chacune des formations sociales historiques à prétention étatique, c'est à dire attachée à un type quelconque de souveraineté territoriale ;

3 - à l'aube de l'époque contemporaine (fin $\mathrm{du} \mathrm{XVIII}^{\mathrm{e}}$ - début du XIX ${ }^{\mathrm{e}}$ siècle), le centre de gravité du peuple juif en Europe (et probablement dans le monde) s'est situé dans une zone dont la Galicie occupait le centre : c'est la matrice historique de la grosse majorité du peuplement juif en Europe de l'Ouest et dans les Amériques, qui s'est produit à la suite des mouvements migratoires des $\mathrm{XIX}^{\mathrm{e}}$ et $\mathrm{XX}^{\mathrm{e}}$ siècles ;

4 - C'est un peuplement essentiellement diasporique, procédant de migrations individuelles : le sens des migrations a été systématiquement dirigé vers le Sud et l'Ouest : d'abord (entre 1780 et 1848) vers la Hongrie, puis (entre 1848 et 1918) vers Vienne ; enfin, depuis les années 1880, vers l'Occident et l'Amérique. Une partie 
importante - probablement majoritaire au $\mathrm{XIX}^{\mathrm{e}}$ siècle - de la migration juive s'est donc confinée à l'Europe centrale elle-même ;

5 - l'origine germanique des langues qu'ils pratiquaient (yiddish ou autres dialectes allemands) apporte une contribution décisive au caractère proprement centre-européen des Juifs : contrairement aux nationalités non germaniques, les Juifs communiquaient plus ou moins directement avec les élites locales allemandes - notamment dans l'Empire des Habsbourg - et disposaient d'un accès direct à la mouvance culturelle dominante ;

6 - de ce point de vue, leur situation rappelle celle qui marque la diaspora allemande et, sous d'autres aspects, celle des Arméniens ou des Tsiganes. Ils s'en distinguent toutefois soit par leur poids démographique, économique et social incomparablement supérieur, soit - par rapport aux Allemands - par l'absence de tout lien politique, culturel, familial ou «identitaire » avec une (ou des) puissance(s) territoriales européenne(s), ou encore - par rapport à tous - par l'altérité radicale qui est le dénominateur commun sous-jacent de leur définition en tant que groupe social.

\section{Position politique dans les formations sociales nationales}

7 Cette présence n'a jamais servi au développement ni d'un nationalisme territorialiste propre, qui se serait traduit par des efforts pour parvenir à la suprématie politique collective dans quelque région que ce soit-comme ce fut la règle pour les autres nationalités depuis le début $\mathrm{du} \mathrm{XIX}^{\mathrm{e}}$ siècle - ni d'une stratégie de domination sociale reposant sur des privilèges exclusifs acquis, comme ce fut le cas pour les classes dominantes traditionnelles (la noblesse d'épée ou de robe) et celles qui leur ont succédé : les élites politiques ou les membres des bureaucraties politiques. Aucun fait historique n'étaie la thèse de l'effort de domination collective sous l'une ou l'autre forme, omniprésente dans le discours de l'antisémitisme contemporain.

8 Les options politiques des Juifs dans les États-nations en formation en Europe centrale à l'époque contemporaine se seront limitées à quatre modèles :

9 - le retrait, non-participation ou séparatisme (à base culturelle, confessionnelle ou socioprofessionnelle), propre aux groupes juifs mal intégrés, mal acceptés ou objets de stigmatisation systématique (comme en Russie, en Pologne, en Lituanie) : le sionisme, le Bund, l'autonomisme (non territorialiste) sous tous leurs avatars en furent les expressions ;

10 - l'alliance stratégique avec tel ou tel autre groupe nationaliste dans un souci d'autodéfense (comme avec les Polonais à Cracovie et ailleurs en Pologne au xIX ${ }^{e}$ siècle ou dans les années 1920, avec les Allemands à Prague au XIX ${ }^{\mathrm{e}}$ siècle également) ;

11 - le nationalisme d'emprunt ou adhésion à des causes nationales représentées par d'autres élites (comme, typiquement, en Hongrie depuis le XIX ${ }^{e}$ siècle jusqu'à nos jours) ;

12 - le soutien d'une autorité supra-nationale (comme dans l'Empire libéral des Habsbourg). Cette dernière option n'a pas toujours été exclusive des trois autres, tant s'en faut, sous la Monarchie bicéphale et au-delà. Elle trouve son illustration paradoxale dans la fameuse boutade sur les «vrais Autrichiens »: si l'on met à part tous les groupes enracinés et titulaires d'une identité spécifique dans l'Empire-les Tyroliens, les Carinthiens, les 
Tchèques, les Moraves, etc. -, il ne reste que les Juifs qui revendiquent d'être des «Autrichiens véritables »...

\section{Conditions idéologiques de l'intégration}

13 Cette boutade souligne aussi le côté précaire du statut des Juifs au sein des groupes nationaux et Etats-nations en formation en Europe centrale, à savoir la tension réelle ou virtuelle qui y a constamment prévalu dans les relations judéo-chrétiennes ; s'il y a eu des moments forts dans la symbiose judéo-chrétienne, ces relations d'intégration, d'acceptation ou d'alliance ont été réversibles, souvent fragiles et tôt ou tard partout fondamentalement remises en cause. Force est de constater que c'est vers la fin de la Monarchie des Habsbourgs (1867-1918) que ces relations ont été le plus proche d'un état d'équilibre et que les nationalismes triomphants de l'entre-deux-guerres ont été partout néfastes à ces relations. Le génocide nazi ne constitue que le point d'orgue externe de ces tensions qui se sont manifestées à l'intérieur de chaque État-nation.

Cette constatation historique conduit à trois observations générales :

15 - fondée à l'origine sur l'altérité radicale, la définition sociale la plus répandue des Juifs dans les pays de l'Europe centrale n'a jamais su éliminer la distance sociale ainsi créée entre Juifs et non Juifs. A classe sociale et à niveau culturel égaux, pareille distance ne s'est maintenue nulle part ailleurs entre agrégats nationaux, culturels ou confessionnels. A cet égard, les pays d'Europe centrale s'opposent aux sociétés occidentales, soit qu'une semblable division ne s'y soit pas conservée (comme dans les pays protestants de l'Europe de l'Ouest et du Nord), soit (comme dans les Amériques) que la gravité de cette division ait été dépassée par d'autres types de divisions, notamment raciales et/ou culturelles (qui opposent les Noirs aux Blancs et aux Jaunes, par exemple). Sous ce rapport, les sociétés d'Europe centrale sont restées à mi-chemin dans un parcours vers la modernité où les distinctions sociales reposent avant tout sur des critères objectifs liés au pouvoir, aux performances, au capital culturel, etc. détenus, et non pas (ou moins) sur le statut attribué et hérité (« de naissance ») comme c'est le cas dans les sociétés traditionnelles. En Europe centrale, contrairement aux sociétés modernes et post-modernes de niveau économique semblable, l'opposition entre Juifs et non Juifs a continué de participer de la définition primaire du statut social ;

16 - comme ailleurs, l'intégration sociale des Juifs (au sens de la disparition ou de la réduction à l'insignifiance de la distance qui les sépare du reste des groupes, à classe sociale ou à niveau culturel égaux) a été mieux garantie sous l'égide des idéologies universalistes: le libéralisme, la démocratie parlementaire, le socialisme, l'humanisme franc-maçon, etc. que sous l'égide des idéologies particularistes, comme le nationalisme ou le fascisme. De la sorte, on observe partout une sur-représentation considérable des Juifs dans les mouvements animés par ces constructions idéologiques. Souvent l'idéologie antisémite (ou du maintien de la distance sociale des Juifs) a pu ainsi s'affubler de références anti-universalistes. C'est le cas typique des idéologies particularistes de l'enracinement (nationalisme de type raciste ou völkisch).

17 - cela n'exclut nullement que des Juifs puissent adopter des nationalismes d'emprunt de type fascisant, à condition qu'ils ne prônent pas l'exclusivisme raciste ou völkisch (comme l'Italie fasciste en offre l'exemple jusqu'en 1938). C'est toutefois un cas limite qui ne se retrouve guère en Europe centrale ; 
18 - le degré de sécularisation des sociétés d'accueil est un critère essentiel de l'intégration des Juifs. Dans la mesure où jadis la distance aux Juifs se définissait partout en termes principalement d'altérité (et d'exclusion) religieuse et où le séparatisme confessionnel a continué de servir de principe de division sociale dans les sociétés multi-confessionnelles, l'affaiblissement de l'influence des Eglises a été une condition sine qua non de l'intégration des Juifs. C'est ainsi qu'elle a le mieux réussi dans les sociétés à multiples églises chrétiennes (comme en Hongrie) ou dans les formations sociales où l'impact clérical était atténué (comme en Bohême) ou même combattu (comme sous le règne des libéraux en Hongrie) ;

19 - la propension à la sécularisation des milieux juifs était la contrepartie indispensable à l'intégration. Selon ce critère, on perçoit partout une dualité d'options. Aux groupes portés à la modernisation intellectuelle - comportant un certain éloignement de l'observance traditionnelle - s'opposent les milieux traditionnels qui résistent souvent à outrance à tout écart par rapport à la tradition. Cette division du judaïsme n'est pas une exception dans tel ou tel pays (comme typiquement en Hongrie où, depuis 1868, il y a trois réseaux institutionnels séparés), mais elle est la règle. C'est la proportion des secteurs traditionalistes et modernistes ainsi que leurs rapports réels avec la tradition qui change historiquement d'un pays à l'autre. L'impact du traditionalisme religieux est fortement rattaché aux facteurs de mobilité géographique et socio-professionnelle. Le traditionalisme religieux et le refus de la sécularisation relèvent aussi sans doute de la nature de la tradition propre : ils sont par exemple plus marqués dans les milieux sous influence hassidique que dans d'autres types d'orthodoxies.

\section{Conditions institutionnelles de l'intégration}

20 On peut les déduire des précédentes remarques. L'intégration fut plus complète et mieux réussie (toujours au sens empirique de la diminution de la distance sociale séparant Juifs et non Juifs à classe sociale et à niveau culturel égaux) lorsque des principes idéologiques universalistes se sont traduits dans les institutions publiques ou, en d'autres termes, lorsque la distance ou la mise à l'écart des Juifs n'étaient pas inscrites dans le fonctionnement des institutions. Quatre champs d'activité collective ont eu sous ce rapport une importance capitale :

21 - le champ politique : les instances politiques à idéologies universalistes ont servi partout de foyers d'intégration (tels les mouvements socialistes et communistes, la francmaçonnerie libérale, les partis radicaux-libéraux, etc.). Dans les pays à champ politique ouvert (comme la Hongrie ou la Bohême), l'intégration politique des Juifs a été la règle ;

22 - la fonction publique : les États-nations ayant tous été dirigés par des élites nationales (le plus souvent issues de la noblesse ou des milieux qui s'y étaient assimilés), la fonction publique restait plus ou moins fermée aux allogènes, surtout aux Juifs. Son degré d'ouverture relative (en Hongrie et en Bohême) est une bonne mesure de l'attitude institutionnelle face à l'intégration des Juifs ;

23 - les réseaux scolaires ouverts (comme en Bohême, en Autriche et en Hongrie) dès l'école primaire ont été des facteurs d'intégration tout à fait essentiels ;

24 - la vie associative spontanée ou organisée par l'État a eu un rôle semblable, en particulier les organisations de jeunesse, les syndicats et associations professionnelles 
(ordre des médecins, barreau), les chambres de commerce et d'industrie ainsi que les instances culturelles locales (cercles de lecture, clubs, associations de bienfaisance, etc.).

Toutefois, il est impossible d'interpréter les disparités dans l'ouverture des institutions d'intégration vis-à-vis des Juifs sans prendre en compte les rapports d'intérêts et de forces qui liaient les classes dominantes et les différentes fractions des élites nationales aux Juifs. L'ouverture relative des institutions en était l'expression en même temps qu'elle reflétait l'importance accordée aux idéologiques universalistes. Là où les Juifs constituaient des alliés utiles (comme pour la bourgeoisie allemande à Prague ou pour la noblesse libérale hongroise avant 1918) au renforcement d'une situation minoritaire, on mettait en place de façon libérale les institutions d'intégration. Là où une telle alliance n'a pas été jugée indispensable, le champ institutionnel est resté divisé en secteurs plus ou moins réservés on interdits aux Juifs.

\section{Conditions socio-économiques de l'intégration}

Toutes choses étant égales par ailleurs, la probabilité de l'intégration s'avérait toujours plus grande là où se développaient les marchés économiques libres que dans des zones de stagnation ou dans des marchés restant sous contrôle étatique. De même, l'intégration avait toujours plus de chances de réussir dans des milieux professionnels issus de l'économie moderne que dans les secteurs économiques traditionnels ou retardés. De part et d'autre, c'est dans le cadre de la dynamique économique de la société industrielle, surtout dans ses phases ascendantes, que les conditions de l'intégration étaient le mieux réunies. Cette proposition générale se maintient en dépit du fait

- qu'il pouvait y avoir entente ou symbiose ou complémentarité harmonieuse entre Juifs et non Juifs dans des secteurs économiques peu dynamiques et traditionnels (entre paysans ou propriétaires terriens d'une part et commerçants juifs d'autre part, comme en Galicie et en Hongrie au XVIII ${ }^{\mathrm{e}}$ et au XIX ${ }^{\mathrm{e}}$ siècle) ;

- que dans la dynamique de l'économie industrielle, des phénomènes de concurrence de classe pouvaient surgir (comme un peu partout entre les bourgeoisies nationale et allogène, notamment juive).

En dépit de ces contre-exemples, ce furent surtout les sociétés urbaines ou des milieux urbanisés (de tous niveaux, du prolétariat à la bourgeoisie), les activités économiques modernes (l'industrie, les banques, le commerce de gros, les professions intellectuelles et libérales) et les groupes professionnels qui s'y rattachaient, qui fournissaient les principaux terrains d'entente et de rapprochement entre Juifs et non Juifs et constituaient les foyers de l'intégration. Les membres de ces milieux « modernisés », issus de part et d'autre d'une mobilité sociale ascendante ou horizontale (par reconversion " économique » des anciennes élites terriennes, ou de fils de la petite bourgeoisie en intellectuels, par exemple) communiaient en effet plus souvent que d'autres dans des idéologies universalistes et/ou passaient, pour atteindre leur statut socio-professionnel, par les mêmes instances de formation (lycées, universités). C'est pour cela que les plus importants foyers d'intégration des Juifs étaient localisés dans les capitales, comme Budapest, Vienne et Prague ainsi que dans d'autres centres de développement de moindre importance.

30 S'il y a des contre-exemples à cette proposition, ils se situent tous plutôt du côté des sociétés d'accueil. Chez les Juifs, il y a partout corrélation univoque entre modernité 
économique (et mobilité sociale correspondante) d'une part, volonté d'intégration par abandon du traditionalisme, d'autre part. La dualité d'attitudes évoquée plus haut a dépendu fortement de la position de chacun sur l'échelle de la modernité économique.

\section{La compensation des désavantages ou la mobilité sociale différentielle}

31 Un mouvement à deux rythmes peut être observé à cet égard. La dualité des milieux juifs définissait des groupes portés à la mobilité et des groupes immobiles. Deux types de déterminations initiales, déjà évoquées, ont joué un grand rôle dans la mobilisation progressive de milieux juifs de plus en plus larges:

- l'état de modernisation économique locale (industrialisation, possibilités commerciales, développement du crédit et des transports). Les milieux juifs ont partout autant ou davantage saisi les chances offertes par le développement économique que les autres groupes (surtout ceux de la majorité nationale, à l'exception de la Bohême). L'immobilisme des masses juives de la Galicie est directement lié au sous-développement prolongé de la région (tardivement et mal desservie par le chemin de fer, etc.) ;

33 - l'impact des migrations géographiques, plus particulièrement l'urbanisation. La mobilité géographique (vers la Hongrie ou vers Vienne par exemple) constituait souvent un acte stratégique de mobilité sociale. Elle sortait des sujets ou des fractions de familles de leur communauté fermée, les soustrayant ainsi à la pesanteur du contrôle social traditionaliste. Elle opérait une sélection des plus aptes à réussir dans l'entreprise de mobilité sociale. Elle contribuait au développement de diverses aptitudes, dont l'aptitude linguistique, favorisant la mobilité sociale.

34 Cela étant, la constatation majeure qui semble se dégager de toutes les études empiriques faites sur ce sujet concerne le surcroît de mobilité de type moderne ou une notable mobilité différentielle dans les champs professionnels issus de l'industrialisation et de la modernisation économique. Cette observation présuppose:- une plus grande force compétitive des Juifs sur les marchés économiques et dans les professions modernes, surtout sur les marchés libres, non protégés par l'État ou non réservés à des clientèles non juives, tels que :

35 - le système bancaire, le commerce de gros, l'industrie, les professions libérales et intellectuelles (où l'on observe une sur-représentation toujours remarquable des Juifs) ;

36 - la scolarisation longue accessible à tous. Les Juifs y ont été non seulement systématiquement sur-représentés mais aussi sur-qualifiés, si l'on en croit les résultats scolaires atteints d'après les études qui ont été menées sur les bacheliers et les étudiants en Hongrie à toutes les époques.

37 - une disposition plus grande à investir tant financièrement qu'intellectuellement dans des domaines nouveaux tels, par exemple, que l'industrie culturelle, la presse, l'édition ou le cinéma.

38 - une meilleure capacité de gestion des capitaux liée aux vertus et compétences «bourgeoises»: effort de prévision, rapport rationnel à l'avenir, adaptabilité aux nouvelles circonstances, calcul rationnel, mode de vie sobre ou sous-consomation relative, stricte discipline de travail, cumul des réserves, etc. 
39 Les raisons de ce surcroît de mobilité et de sur-qualification sont multiples. Sans doute y trouve-t-on des aspects d'un certain rationalisme culturel, lié à la pratique religieuse (alphabétisation historiquement précoce, pratique fondée sur la lecture et sur l'interprétation), et des dispositions économiques anciennes se rattachant aux métiers auxquels les Juifs étaient confinés et astreints avant l'émancipation (commerce, trafic d'argent, etc.). Il y a surtout le besoin de compenser les désavantages socio-économiques subis et la faible compétitivité des concurrents sociaux;

40 - les performances supérieures et le cumul de capitaux économiques et culturels répondent directement à la précarité du statut social des Juifs en Europe centrale ;

41 - certains types de performances symboliques (objectivées par exemple dans les résultats scolaires ou dans des diplômes supérieurs) étaient souvent directement motivés parce qu'ils favorisaient la mobilité sur l'échelle des statuts sociaux, c'est à dire l'intégration dans les classes moyennes ou dans les élites chrétiennes ;

42 - ce déséquilibre en faveur des Juifs des rapports de forces sur les marchés professionnels modernes - effet de la surcompensation des désavantages initiaux - était un des éléments constants des discours antisémites, exprimant les ressentiments des perdants dans des situations de concurrence ouverte entre fractions juives et non juives dans des classes sociales et des secteurs d'activité divers.

\section{Les fonctions sociales des juifs dans les sociétés d'Europe centrale}

43 On peut identifier un certain nombre de fonctions remplies surtout par les Juifs pour le compte des sociétés nationales, qui distinguent leurs performances collectives de celles des autres groupes.

441 - Médiation de biens culturels et économiques ainsi que de modèles de comportement ressortissant à la modernité occidentale. Cette fonction-essentielle dans les pays culturellement dominés et à développement économique tardif de l'Europe centrale - s'est déployée dans des domaines très variés : les sciences de pointe, les arts d'avant-garde, la presse de masse, l'importation d'idéologies politiques, la gestion des entreprises, l'instauration de nouvelles conduites démographiques (malthusianisme), intellectuelles (multi-linguisme, études à l'étranger), pédagogiques (sans violence ou à violence symbolique), etc.

45 Les conditions de réalisation de cette fonction relevaient du caractère diasporique $d u$ peuplement juif (s'étendant jusqu'aux pays occidentaux), d'une propension à la surinformation (dont un aspect essentiel se rattache aux réflexes collectifs de défense de soi), d'une capacité d'information (liée entre autres au plurilinguisme) et de l'absence de barrages culturels devant la modernité occidentale dans le judaïsme réformé (pas de poussées anti-modernistes, comme dans le catholicisme ultramontain par exemple...). L'accomplissement de cette fonction est tout à fait spécifique de l'Europe centrale : elle y est plus forte que dans les autres régions de l'Europe.

2 - Création ou contribution à la création des secteurs de l'économie moderne. On observe dans la modernisation de chaque économie nationale des secteurs entiers (pas toujours identiques) au développement desquels la contribution des Juifs fut prépondérante. Ce sont le plus souvent le haut négoce en produits finis, le journalisme de 
masse et la presse culturelle, l'industrie textile, le réseau bancaire, l'industrie culturelle privée (cinéma, etc.) et les diverses branches des professions intellectuelles-libérales (médecine partout, droit en Hongrie, etc.). Ces secteurs se sont souvent développés à partir de l'exploitation économique d'innovations techniques majeures. Le poids économique relatif des Juifs n'a probablement été nulle part ailleurs au monde aussi important avant le génocide nazi qu'à Budapest, Vienne, Lwov, Varsovie ou à Czernovitz.

473 -Contribution à l'invention des systèmes d'idées et de pratiques intellectuelles de la modernité dans les champs politique, culturel-esthétique, littéraire, philosophique, scientifique, etc. Le poids intellectuel des Juifs n'a été probablement nulle part ailleurs aussi décisif que dans les centres intellectuels de ces régions (surtout à Vienne) : qu'on pense aux oeuvres de Freud, de Kafka, de Wittgenstein, etc.

\section{Autres perspectives}

Il faut évoquer en conclusion deux autres aspects de la présence juive qui déborde le cadre de l'Europe centrale: l'antisémitisme et les processus d'assimilation. Loin de s'exclure mutuellement, ils ont tous deux trouvé des points forts en Europe centrale. Mais cette question est l'objet d'une autre étude.

\section{AUTEUR}

\section{VICTOR KARADY}

Victor KARADY est directeur de recherche au CNRS. Ce texte a fait l'objet d'une communication au colloque sur l'Europe centrale, qui s'est tenu à Varsovie en septembre 1990. 